\title{
Homotopy Perturbation Method for Thin Film Flow and Heat Transfer over an Unsteady Stretching Sheet with Internal Heating and Variable Heat Flux
}

\author{
I-Chung Liu' ${ }^{1}$ and Ahmed M. Megahed ${ }^{2}$ \\ ${ }^{1}$ Department of Civil Engineering, National Chi Nan University, 1 University Road, Puli, \\ Nantou 545, Taiwan \\ ${ }^{2}$ Department of Mathematics, Faculty of Science, Benha University, Benha 13511, Egypt
}

Correspondence should be addressed to I-Chung Liu, icliu@ncnu.edu.tw

Received 6 June 2012; Accepted 22 July 2012

Academic Editor: Subhas Abel

Copyright (C) 2012 I.-C. Liu and A. M. Megahed. This is an open access article distributed under the Creative Commons Attribution License, which permits unrestricted use, distribution, and reproduction in any medium, provided the original work is properly cited.

\begin{abstract}
We have analyzed the effects of variable heat flux and internal heat generation on the flow and heat transfer in a thin film on a horizontal sheet in the presence of thermal radiation. Similarity transformations are used to transform the governing equations to a set of coupled nonlinear ordinary differential equations. The obtained differential equations are solved approximately by the homotopy perturbation method (HPM). The effects of various parameters governing the flow and heat transfer in this study are discussed and presented graphically. Comparison of numerical results is made with the earlier published results under limiting cases.
\end{abstract}

\section{Introduction}

The flow and heat transfer characteristics inside thin films have attracted the interest of many researchers. This is due to their several applications in engineering such as foodstuff processing, reactor fluidization, wire and fiber coating, cooling of metallic plates, drawing of a polymer sheet, aerodynamic extrusion of plastic sheets, continuous casting, rolling, annealing, and tinning of copper wires. In the extrusion process, this understanding is crucial for maintenance of the surface quality of the extrudate. The coating process requires a smooth surface for the best product appearance and for such properties as low friction, transparency, and strength. As the quality of product in the extrusion processes depends considerably on the flow and heat transfer characteristics of a thin liquid film over a stretching sheet, analysis of momentum and heat transfer in such processes is essential. Crane [1] gave an exact similarity solution for the problem of steady two-dimensional boundary layer flow caused by the stretching of a sheet which moves in its own plane with velocity varying 
linearly with distance from a fixed point. The problem of flow, heat, and mass transfer over a stretching sheet in the presence of suction or blowing was examined by P. S. Gupta and A. S. Gupta [2]. Grubka and Bobba [3] analytically considered the stretching sheet problem for a surface moving with a linear velocity and with a variable surface temperature. Ali [4] investigated flow and heat transfer characteristics due to a stretching surface with velocity and temperature distributions being of a power-law type. Vajravelu [5] presented analytical and numerical schemes to solve the problem of convective heat transfer over a stretching sheet with suction and blowing. Pop and $\mathrm{Na}$ [6] analyzed the unsteady flow past a wall which starts impulsively to stretch from rest. They found that the unsteady flow solution would approach the steady flow situation after long passage of time. Mahapatra and Gupta [7] analyzed stagnation-point flow towards a stretching surface in the presence of free stream velocity.

In all the above studies, the steady or unsteady boundary layer equation is considered and the boundary conditions are prescribed at the stretching sheet and on the fluid at infinity. Wang [8] first studied the flow problem within a thin liquid film on an unsteady stretching surface, where the similarity transformation was used to transform the governing partial differential equations to a nonlinear ordinary differential equation with an unsteadiness parameter. Andersson et al. [9] studied the flow problem of a power-law fluid film on an unsteady stretching surface considered by Wang [8]. Later Andersson et al. [10] extended Wang's problem [8] to the case of heat transfer. Dandapat et al. [11] investigated the effect of the thermocapillarity on the flow and heat transfer in a thin liquid film over an unsteady stretching sheet. Chen [12] numerically studied the heat transfer occurring in a thin liquid film of a power-law fluid over an unsteady stretching sheet. Wang [13] presented exact analytical solutions to the unsteady momentum and heat transfer problem within a liquid film whose motion is caused solely by the linear stretching of a horizontal elastic sheet. The HAM solutions for the flow behavior of a power-law fluid due to an unsteady stretching surface in the case of finite thickness were presented by Wang and Pop [14]. The effect of variable thermal properties on flow and heat transfer in a liquid film over an unsteady stretching sheet was analyzed by Dandapat et al. [15] for viscous Newtonian fluids and by Mahmoud and Megahed [16] for power-law fluids. Noor and Hashim [17] investigated the effects of thermocapillarity and a magnetic field in a thin liquid film on an unsteady elastic stretching sheet. A recent study on boundary layer behavior for flow over a semi-infinite unsteady stretching surface was done by Abd El-Aziz [18]. He studied the effect of Hall currents on the flow and heat transfer of an electrically conducting fluid over an unsteady stretching surface in the presence of a strong magnetic field. Very recently, Aziz et al. [19] analyzed the influence of internal heat generation on flow and heat transfer in a thin liquid film on an unsteady stretching sheet.

To the best of the authors' knowledge, there are very few papers, with the exception of Megahed [20] and Liu and Megahed [21], considering the heat transfer aspects in a liquid film flow over an unsteady stretching sheet subject to variable surface heat flux conditions. Motivated by above literature, the purpose of the present study is to investigate the heat generation effect on thin film flow and heat transfer over an unsteady stretching surface in the presence of variable heat flux and thermal radiation.

\section{Formulation of the Problem}

Consider the unsteady flow of a Newtonian fluid in a thin liquid film over a stretching surface. The elastic sheet issues from a narrow slit at the origin of a Cartesian coordinate 
system as shown in Figure 1. The continuous surface aligned with the $x$-axis at $y=0$ moves in its own plane with a velocity $U(x, t)$. A thin liquid film of uniform thickness $h(t)$ lies on the horizontal surface. The surface heat flux $q(x, t)$ at the stretching sheet varies with the power of distance $x$ from the slit and with the inverse power of time factor $t$ as

$$
q=-\kappa \frac{\partial T}{\partial y}=-T_{0} \frac{d x^{2}}{\beta(1-a t)^{2}},
$$

where $\kappa$ is the thermal conductivity, $T_{0}$ is a (positive or negative; heating or cooling) reference temperature, $d$ is a constant, and $a \ll t^{-1}$ is a positive constant with dimension reciprocal time. The governing time-dependent boundary layer equations for mass, momentum, and energy conservation are given by

$$
\begin{gathered}
\frac{\partial u}{\partial x}+\frac{\partial v}{\partial y}=0 \\
\frac{\partial u}{\partial t}+u \frac{\partial u}{\partial x}+v \frac{\partial u}{\partial y}=\frac{\mu}{\rho} \frac{\partial^{2} u}{\partial y^{2}} \\
\frac{\partial T}{\partial t}+u \frac{\partial T}{\partial x}+v \frac{\partial T}{\partial y}=\frac{\kappa}{\rho c_{p}} \frac{\partial^{2} T}{\partial y^{2}}-\frac{1}{\rho c_{p}} \frac{\partial q_{r}}{\partial y}+\frac{Q}{\rho c_{p}},
\end{gathered}
$$

where $u$ and $v$ are the velocity components along the $x$ and $y$ directions, respectively. $\rho$ is the fluid density, $T$ is the temperature of the fluid, $t$ is the time, $\mu$ is the viscosity of the fluid, $q_{r}$ is the radiative heat flux, and $c_{p}$ is the specific heat at constant pressure. The $Q$ term is the heat generated $(>0)$ or absorbed $(<0)$ per unit volume and is defined as [19]

$$
Q=\frac{\kappa \rho U}{\mu x} B^{*}\left(T-T_{0}\right)
$$

where $B^{*}$ is the temperature-dependent heat generation/absorption. That is, $B^{*}$ is positive in the case of generation of heat and negative in the case of absorption of heat within the fluid system.

The appropriate boundary conditions for the present problem are

$$
\begin{gathered}
u=U(x, t), \quad v=0, \quad-\kappa \frac{\partial T}{\partial y}=q(x, t) \quad \text { at } y=0, \\
\frac{\partial u}{\partial y}=\frac{\partial T}{\partial y}=0 \quad \text { at } y=h(t), \\
v=\frac{d h}{d t} \quad \text { at } y=h,
\end{gathered}
$$

where $U(x, t)$ is the surface velocity of the stretching sheet defined later, $h$ is the thickness of the liquid film. The flow is caused by stretching the elastic surface at $y=0$ such that the continuous sheet moves in the $x$-direction with the velocity [8]:

$$
U=\frac{b x}{1-a t}
$$




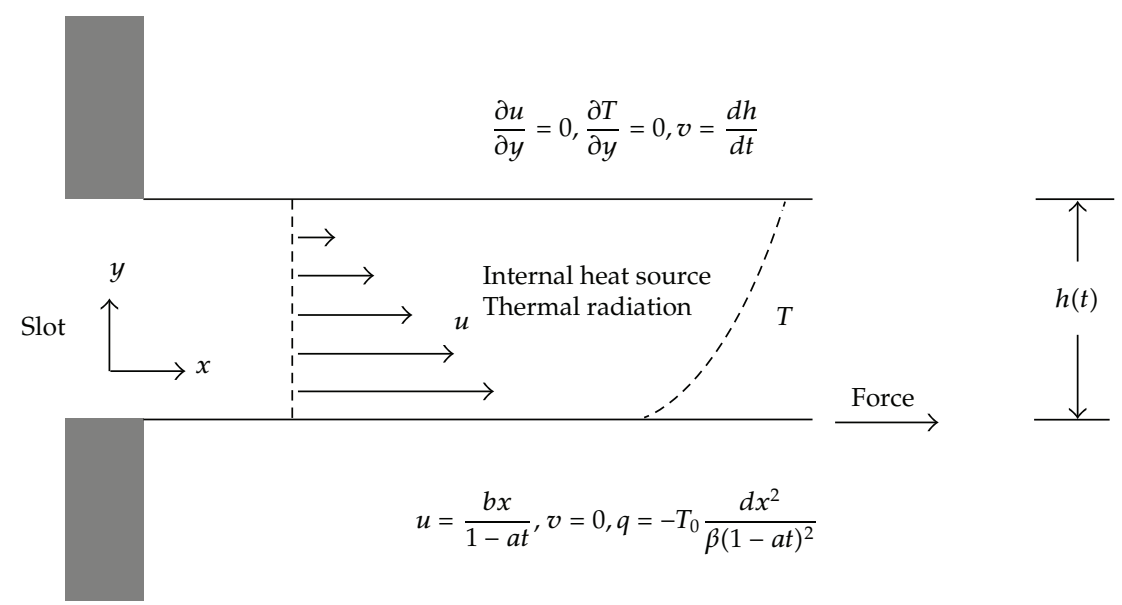

Figure 1: Schematic diagram of the physical configuration.

where $a$ and $b$ are positive constants with dimension(time $)^{-1}$, such that $a<1 / t$ is required so as to allow the stretching of the elastic sheet.

The radiative heat flux $q_{r}$ is employed according to Rosseland approximation [22] such that

$$
q_{r}=-\frac{4 \sigma^{*}}{3 k^{*}} \frac{\partial T^{4}}{\partial y}
$$

where $\sigma^{*}$ is the Stefan-Boltzmann constant, $k^{*}$ is the mean absorption coefficient. Following Raptis [23], we assume that the temperature differences within the flow are small such that $T^{4}$ may be expressed as a linear function of the temperature. Expanding $T^{4}$ in a Taylor series about $T_{0}$ and neglecting higher-order terms, we have

$$
T^{4} \cong 4 T_{0}^{3} T-3 T_{0}^{4} \text {. }
$$

The special form of the surface velocity (2.7) and the surface heat flux (2.1) allows the system of partial differential equations (2.3) and (2.4) to be transformed into a system of coupled nonlinear ordinary differential equations by using the following similarity transformation:

$$
\begin{gathered}
\eta=\left(\frac{b}{\mu / \rho}\right)^{1 / 2}(1-a t)^{-1 / 2} \beta^{-1} y, \\
u=b x(1-a t)^{-1} f^{\prime}(\eta), \\
v=-\left(\frac{\mu b}{\rho}\right)^{1 / 2}(1-a t)^{-1 / 2} \beta f(\eta), \\
T=T_{0}-T_{0}\left(\frac{d x^{2}}{\kappa \sqrt{\rho b / \mu}}\right)(1-a t)^{-3 / 2} \theta(\eta), \\
\theta=\frac{T_{0}-T}{\Delta T}, \quad \Delta T=T_{0}\left(\frac{d x^{2}}{\kappa \sqrt{\rho b / \mu}}\right)(1-a t)^{-3 / 2},
\end{gathered}
$$


where $\beta$ is yet an unknown constant denoting the dimensionless thin film thickness, defined by [17]

$$
\beta=\left(\frac{b}{\mu / \rho}\right)^{1 / 2}(1-a t)^{-1 / 2} h(t)
$$

Using (2.10), the mathematical problem defined in (2.2)-(2.4) are then transformed into the following set of ODEs:

$$
\begin{gathered}
f^{\prime \prime \prime}+r\left[f f^{\prime \prime}-f^{\prime 2}-S f^{\prime}-\frac{S}{2} \eta f^{\prime \prime}\right]=0, \\
\left(\frac{1+R}{\operatorname{Pr}}\right) \theta^{\prime \prime}+r\left[f \theta^{\prime}-2 f^{\prime} \theta-\frac{3}{2} S \theta-\frac{S}{2} \eta \theta^{\prime}+\frac{B^{*}}{\operatorname{Pr}} \theta\right]=0,
\end{gathered}
$$

subject to the boundary conditions:

$$
\begin{gathered}
f(0)=0, \quad f^{\prime}(0)=1, \quad \theta^{\prime}(0)=-1, \\
f^{\prime \prime}(1)=0, \quad \theta^{\prime}(1)=0, \\
f(1)=\frac{S}{2},
\end{gathered}
$$

where primes denote differentiation with respect to $\eta, S=a / b$ is the unsteadiness parameter, $\operatorname{Pr}=\mu c_{p} / \kappa$ is the Prandtl number, $\gamma=\beta^{2}$ is the dimensionless film thickness, and $R=$ $16 \sigma^{*} T_{0}^{3} / 3 k^{*} \kappa$ is the radiation parameter.

The physical quantities of interest are the skin-friction coefficient $C_{f}$, and the local Nusselt number $\mathrm{Nu}_{x}$ which are defined as

$$
\begin{aligned}
& C_{f}=\frac{-2}{\beta} f^{\prime \prime}(0) \operatorname{Re}_{x}^{-1 / 2}, \\
& N u_{x}=(1+R) \frac{\operatorname{Re}_{x}^{1 / 2}}{\beta \theta(0)},
\end{aligned}
$$

where $\operatorname{Re}=\rho U x / \mu$ is the local Reynolds number. We note that reduced local Nusselt number employed in (2.18) contains two terms: one comes from the usual Fourier's law of conduction term and the other is thermal radiation flux term. A simple and frequently used representation for above two quantities is the reduced skin-friction coefficient and reduced Nusselt number, which have been defined as

$$
\begin{aligned}
& \frac{C_{f} \beta \operatorname{Re}_{x}^{1 / 2}}{2}=-f^{\prime \prime}(0), \\
& \frac{N u_{x}}{1+R} \operatorname{Re}_{x}^{-1 / 2} \beta=\frac{1}{\theta(0)} .
\end{aligned}
$$




\section{Solution Procedure Using HPM}

In this section, we implement HPM to obtain the approximate solution for the thin film flow and heat transfer over an unsteady stretching surface with internal heating in the presence of variable heat flux and thermal radiation. Now according to HPM, we can construct the following simple homotopy for functions $f$ and $\theta$ as

$$
\begin{gathered}
f^{\prime \prime \prime}+q r\left[f f^{\prime \prime}-f^{\prime 2}-S f^{\prime}-\frac{S}{2} \eta f^{\prime \prime}\right]=0, \\
\theta^{\prime \prime}+q\left(\frac{r \operatorname{Pr}}{1+R}\right)\left[f \theta^{\prime}-2 f^{\prime} \theta-\frac{3}{2} S \theta-\frac{S}{2} \eta \theta^{\prime}+\frac{B^{*}}{\operatorname{Pr}} \theta\right]=0,
\end{gathered}
$$

where $q \in[0,1]$ is an embedding parameter. We use it to expand the solutions in the form:

$$
f(\eta)=f_{0}+q f_{1}+q^{2} f_{2}+\cdots, \quad \theta(\eta)=\theta_{0}+q \theta_{1}+q^{2} \theta_{2}+\cdots .
$$

Substituting from (3.2) into (3.1) and equating the terms to the identical powers of $q$, we can obtain a system of $n+1$ linear ODEs. Assuming $n=2$ the system becomes

$$
\begin{gathered}
f_{0}^{\prime \prime \prime}=0, \quad f_{0}(0)=0, \quad f_{0}^{\prime}(0)=1, \quad f_{0}^{\prime \prime}(0)=\alpha, \\
\theta_{0}^{\prime \prime}=0, \quad \theta_{0}(0)=\varepsilon, \quad \theta_{0}^{\prime}(0)=-1, \\
f_{1}^{\prime \prime \prime}=r\left(\frac{S}{2} \eta-f_{0}\right) f_{0}^{\prime \prime}+\gamma\left(f_{0}^{\prime}+S\right) f_{0}^{\prime}, \quad f_{1}(0)=f_{1}^{\prime}(0)=f_{1}^{\prime \prime}(0)=0, \\
\theta_{1}^{\prime \prime}=\left(\frac{\gamma \operatorname{Pr}}{1+R}\right)\left[\left(\frac{S}{2} \eta-f_{0}\right) \theta_{0}^{\prime}+\left(\frac{3}{2} S+2 f_{0}^{\prime}-\frac{B^{*}}{\operatorname{Pr}}\right) \theta_{0}\right], \quad \theta_{1}(0)=\theta_{1}^{\prime}(0)=0, \\
f_{2}^{\prime \prime \prime}=-\gamma f_{1} f_{0}^{\prime \prime}+\gamma\left(\frac{S}{2} \eta-f_{0}\right) f_{1}^{\prime \prime}+\gamma f_{0}^{\prime} f_{1}^{\prime}+\gamma\left(S+f_{0}^{\prime}\right) f_{1}^{\prime}, \quad f_{2}(0)=f_{2}^{\prime}(0)=f_{2}^{\prime \prime}(0)=0, \\
\theta_{2}^{\prime \prime}=\left(\frac{\gamma \operatorname{Pr}}{1+R}\right)\left[\left(-f_{0} \theta_{1}^{\prime}+\frac{S}{2} \eta-f_{1} \theta_{1}^{\prime}+\left(\frac{3}{2} S+2 f_{0}^{\prime}-\frac{B^{*}}{\operatorname{Pr}}\right) \theta_{1}\right)+2 f_{1}^{\prime} \theta_{0}\right], \quad \theta_{2}(0)=\theta_{2}^{\prime}(0)=0,
\end{gathered}
$$

for some constants $\alpha=f^{\prime \prime}(0), \varepsilon=\theta(0)$ and $\gamma$. The solutions of the systems (3.3) are

$$
\begin{aligned}
& f_{0}(\eta)=\frac{\alpha}{2} \eta^{2}+\eta \\
& \theta_{0}(\eta)=-\eta+\varepsilon \\
& f_{1}(\eta)=\frac{1}{240} \gamma \eta^{3}\left(5 S(8+3 \alpha \eta)+2\left(20+5 \alpha \eta+\alpha^{2} \eta^{2}\right)\right) \\
& \theta_{1}(\eta)=\frac{r \eta^{2}}{24(1+R)}\left(-\eta\left(-4 B^{*}+\operatorname{Pr}(4+8 S+3 \alpha \eta)\right)+2 \varepsilon\left(-6 B^{*}+\operatorname{Pr}(12+9 S+4 \alpha \eta)\right)\right)
\end{aligned}
$$




$$
\begin{aligned}
& f_{2}(\eta)=\frac{1}{40320}\left(672 S(1+S)+14 \alpha(4+3 S(4+5 S)) \eta+4 \alpha^{2}(-2+9 S) \eta^{2}-\alpha^{3} \eta^{3}\right) \\
& \theta_{2}(\eta)=\frac{r^{2} \eta^{4}}{10080(1+R)^{2}}\{ \eta\left(-84\left(\operatorname{Pr}^{2}\left(-1+S+6 S^{2}\right)+B^{* 2}+5 \operatorname{Pr}\left(1+R+S+R S-S B^{*}\right)\right)\right. \\
&\left.-7 \alpha \operatorname{Pr}\left(7(1+R)(2+3 S)+\operatorname{Pr}(-8+29 S)-10 B^{*}\right) \eta-18 \alpha^{2} \operatorname{Pr}(1+R) \eta^{2}\right) \\
&+7 \varepsilon\left(15\left(5 \operatorname{Pr}^{2} S(4+3 S)+4 \eta^{2}+8 \operatorname{Pr}\left(1+R+S+R S-B^{*}-2 S B^{*}\right)\right)\right. \\
&+6 \alpha \operatorname{Pr}\left(2(1+R)(2+3 S)+\operatorname{Pr}(8+21 S)-10 B^{*}\right) \eta \\
&\left.\left.+4 \alpha^{2} \operatorname{Pr}(1+2 \operatorname{Pr}+R) \eta^{2}\right)\right\} .
\end{aligned}
$$

By the same procedure, we can obtain the other components of the solutions $f(\eta)$ and $\theta(\eta)$. Having found $f_{i}$ and $\theta_{i}, i=0,1,2, \ldots, 8$, the approximate solutions $f(\eta)$ and $\theta(\eta)$ are as follows:

$$
f(\eta) \cong \sum_{i=0}^{8} f_{i}, \quad \theta(\eta) \cong \sum_{i=0}^{8} \theta_{i}
$$

The constants $\alpha, \varepsilon$, and $\gamma$ are given using the outer boundary conditions $f^{\prime \prime}(1)=0, \theta^{\prime}(1)=0$, and $f(1)=S / 2$ simultaneously. We note that all the obtained numerical results are performed using symbolic software MATHEMATICA version 6.

\section{Results and Discussion}

As shown in (2.12)-(2.13) and boundary condition (2.14)-(2.16), the governing parameters for the problem under study are the unsteadiness parameter $S$, heat generation parameter $B^{*}$, Prandtl number Pr, and radiation parameter $R$. Wang [8] conducted the analysis of the flow problem and drew his conclusion that the solution can be obtained only if $S \in[0,2]$ and no solution was found if $S$ lies outside of [0,2]. When $S=0$, Crane's [1] situation of infinite fluid layer is recovered $\beta \rightarrow \infty$, and the infinitesimal liquid film case $\beta \rightarrow 0$ is encountered if $S=2$. Therefore, the dimensionless film thickness $\beta$ is a decreasing function of the unsteadiness parameter $S$, which can be found in Wang's [8] paper and there is no need to duplicate. We note that the film thickness depend on magnetic field and rotation, as depicted in the work by Bandapat and Layek [24] for film coating problem. Moreover, the wall shear stress $-f^{\prime \prime}(0)$ is 1 at $S=0$, and subsequently increases to its maximum 1.231 near $S=1.11$, and then decreases to zero as $S$ reaches the value of 2 . That is, not only the variation of $-f^{\prime \prime}(0)$ is a nonmonotonic function of $S$ but also the velocity profile.

The temperature profiles for varying values of $S$ are depicted in Figure 2 with the parameter $B^{*}=0.5, R=0.5$, and $\operatorname{Pr}=1$. The temperature profiles are observed to raise as the unsteadiness parameter $S$ increases. In addition, both the wall temperature $\theta(0)$ and the free surface temperature $\theta(\beta)$ show the same dependence of $S$ on temperature profile.

Figure 3 shows that when the heat generation parameter $B^{*}$ increases, the temperature increases, which causes the heat transfer rate or local Nusselt number $1 / \theta(0)$ to decrease accordingly, implying the slowdown of the rate of cooling for the thin film flow. On physical 


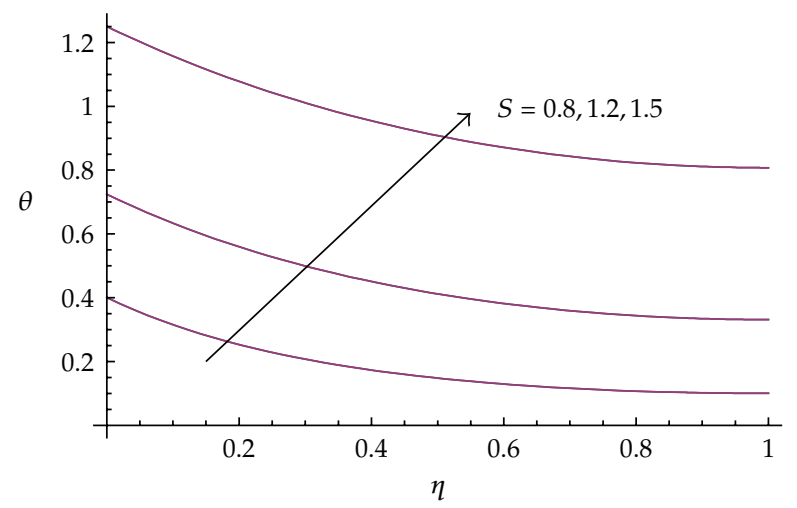

Figure 2: The behavior of the temperature distribution for various values of $S$ with $B^{*}=0.5, R=0.5$, and $\operatorname{Pr}=1$.

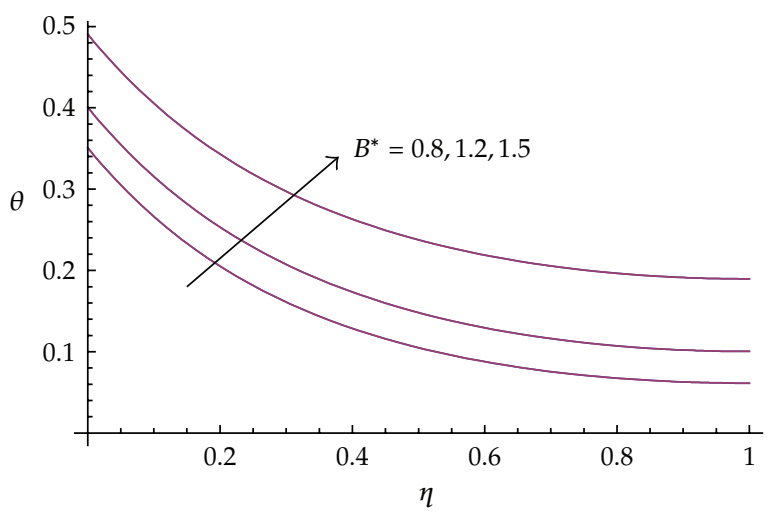

Figure 3: The behavior of the temperature distribution for various values of $B^{*}$ with $S=0.8, R=0.5$, and $\operatorname{Pr}=1$.

ground the increase of internal heat parameter reflects to the enhancement of heat generation within the fluid system, thus the temperature profiles rise correspondingly. This reduces the cooling rate between the stretching sheet and the liquid film. Figure 4 illustrates the influence of Prandtl number on the temperature profiles with fixed parameter $S=0.8, R=0.5$ and $B^{*}=0.5$. From this figure, one can observe that increasing the Prandtl number Pr causes a rise in the rate of heat transfer $1 / \theta(0)$ from the flow to the surface. This will speed up the rate of cooling of the thin film flow as depicted in Figure 4, which is the typical effect of Prandtl number. As the Prandtl number raises, both the wall temperature and the free surface temperature are found to decrease.

Figure 5 shows the fluid temperature distribution plotted against the similarity parameter $\eta$ for different values of $R=0,0.5$ and 2 with $S=0.8, \operatorname{Pr}=3$, and $B^{*}=0.5$. It can be seen that the temperature increases with an increase in the radiation parameter $R$, which leads to a fall in the rate of cooling for the thin film flow. Apparently when the amount of heat radiated within the fluid system due to thermal radiation effect enhances, the temperature profile, wall temperature as well as the free surface temperature should be higher than those when no radiation effect is considered $(R=0)$. 


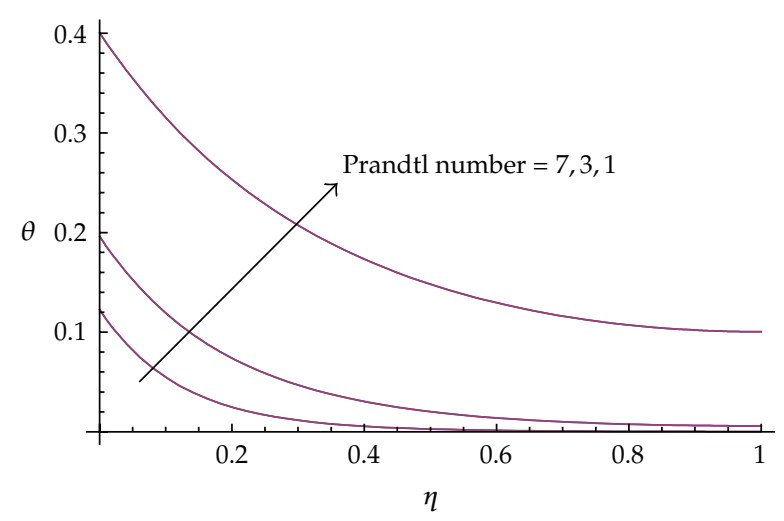

Figure 4: The behavior of the temperature distribution for various values of $\operatorname{Pr}$ with $S=0.8, R=0.5$, and $B^{*}=0.5$.

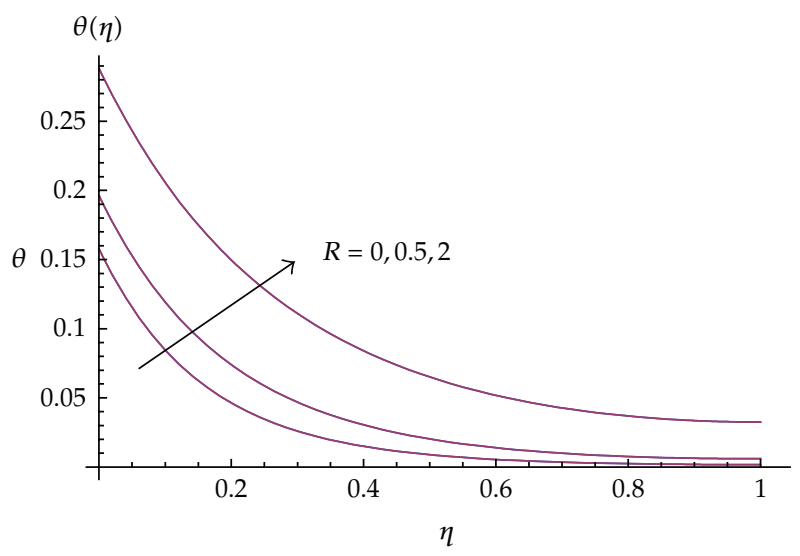

Figure 5: The behavior of the temperature distribution for various values of $R$ with $S=0.8, \operatorname{Pr}=3$, and $B^{*}=0.5$.

The physical implication of Figures 3-5 can be qualitatively recognized by integrating (2.13) from zero to unity and using the boundary conditions (2.14)-(2.16) to yield

$$
\frac{1+R}{\operatorname{Pr}}=r\left[3 \int_{0}^{1} f^{\prime} \theta d \eta+\left(S-\frac{B^{*}}{\operatorname{Pr}}\right) \int_{0}^{1} \theta d \eta\right] .
$$

The boundary layer approximation suggests that velocity function satisfy the condition $0 \leq$ $f^{\prime} \leq 1$, and the physically admissible solution for temperature function requires the condition $\theta \geq 0$. This makes the two integrals inside the square bracket in (4.1) positive definite. It is clear form (2.12) that the velocity $f^{\prime}$ and film thickness $\gamma$ (or $\beta$ ) remain unchanged for fixed value of unsteadiness parameter $S$ [8]. Thus, when $B^{*}=0$ and fixed $R$, the temperature profile should be reduced to meet the requirement of (4.1) for increasing value of Pr. Consequently, the lessening result of thermal boundary layer thickness due to increasing $\operatorname{Pr}$ (or reducing thermal diffusivity effect) is assured. Similarly, with fixed $S$ and $\operatorname{Pr}$ the temperature profiles rise when the value of $R$ enhances. 
Table 1: Comparison of values of skin friction coefficient $-f^{\prime \prime}(0)$ and $\beta=\gamma^{1 / 2}$ using HPM.

\begin{tabular}{lcccc}
\hline$S$ & \multicolumn{2}{c}{$-f^{\prime \prime}(0)$} & \multicolumn{2}{c}{$\beta=\gamma^{1 / 2}$} \\
\hline 0.4 & Wang [13] & Present work & Wang [13] & Present study \\
0.6 & 6.69912 & 6.699108 & 5.12249 & 5.12246 \\
0.8 & 3.74233 & 3.742316 & 3.13125 & 3.131252 \\
1 & 2.68094 & 2.680943 & 2.15199 & 2.151989 \\
1.2 & 1.97238 & 1.972317 & 1.54362 & 1.543622 \\
1.4 & 1.442631 & 1.442621 & 1.12778 & 1.127779 \\
1.6 & 1.012784 & 1.012735 & 0.821032 & 0.821033 \\
1.8 & 0.642397 & 0.642368 & 0.576173 & 0.576171 \\
\hline
\end{tabular}

Table 2: Values for $-f^{\prime \prime}(0), 1 / \theta(0)$ and $\gamma$ for different values of $S, B^{*}, \operatorname{Pr}$, and $R$ using HPM.

\begin{tabular}{ccccccc}
\hline$S$ & $B^{*}$ & $P r$ & $R$ & $-f^{\prime \prime}(0)$ & $1 / \theta(0)$ & $\gamma$ \\
\hline 0.8 & 0.5 & 1 & 0.5 & 2.68096 & 2.49649 & 4.63106 \\
1.2 & 0.5 & 1 & 0.5 & 1.44260 & 1.38111 & 1.27185 \\
1.5 & 0.5 & 1 & 0.5 & 0.821843 & 0.799913 & 0.48045 \\
0.8 & 0 & 1 & 0.5 & 2.68096 & 2.85021 & 4.63106 \\
\hline 0.8 & 0.5 & 1 & 0.5 & 2.68096 & 2.49649 & 4.63106 \\
0.8 & 1 & 1 & 0.5 & 2.68096 & 2.03864 & 4.63106 \\
\hline 0.8 & 0.5 & 1 & 0.5 & 2.68096 & 2.49649 & 4.63106 \\
0.8 & 0.5 & 3 & 0.5 & 2.68096 & 5.0963 & 4.63106 \\
0.8 & 0.5 & 7 & 0.5 & 2.68096 & 8.1543 & 4.63106 \\
0.8 & 0.5 & 3 & 0 & 2.68096 & 6.34069 & 4.63106 \\
0.8 & 0.5 & 3 & 0.5 & 2.68096 & 5.0963 & 4.63106 \\
0.8 & 0.5 & 3 & 2 & 2.68096 & 3.47046 & 4.63106 \\
\hline
\end{tabular}

Table 1 provides the comparison of present results with those of Wang [13]. Without any doubt, we can claim that the comparison is in excellent agreement under some limiting cases $R=0$ and $B^{*}=0$. Table 2 presents the values of reduced skin friction coefficient $-f^{\prime \prime}(0)$ and reduced Nusselt number $1 / \theta(0)$ for several parameters governing the flow and heat transfer aspects. From this Table, we observe that as the unsteadiness parameter $S$ increases, the values of reduced skin friction coefficient and reduced Nusselt number are found to be degraded, which will cause a loss in the rate of cooling for the liquid film flow. Likewise, increasing the Prandtl number Pr leads to an increase in the value of Nusselt number, implying a raise in the rate of cooling for the thin film flow. But, the reverse is true for the radiation parameter $R$. It is noted that the liquid film thickness shortens with the increase of unsteadiness parameter $S$, as observed by Wang [8] for constant surface temperature case and Wang [13] for prescribed variable surface temperature case. Since the momentum equation (2.12) does not contain $\operatorname{Pr}, S$, and $R$, the reduced skin friction coefficient is supposed to be independent on the three values, as is evident in Table 2.

\section{Conclusions}

In this paper, the influence of internal heat generation on the thin film flow and heat transfer on an unsteady stretching sheet in the presence of variable heat flux and thermal radiation has been analyzed successfully by means of homotopy perturbation method (HPM). A 
qualitative argument for the effects of Prandtl number and thermal radiation on temperature variations is provided through the consequence of a simple integration procedure. The present study reveals that the temperature dependent heat generation parameter has no substantial effect on the velocity, but it has a great effect on the temperature and hence on the rate of heat transfer. The presence of internal heating and thermal radiation produces a fall in the cooling rate for the thin film flow. But, the reverse effect is true for the Prandtl number. Also, the high values of unsteadiness parameter lead to a decrease in the rate of cooling for the thin film flow.

\section{References}

[1] L. J. Crane, "Flow past a stretching plate," Zeitschrift für Angewandte Mathematik und Physik, vol. 21, no. 4, pp. 645-647, 1970.

[2] P. S. Gupta and A. S. Gupta, "Heat and mass transfer on a stretching sheet with suction or blowing," Canadian Journal of Chemical Engineers, vol. 55, pp. 744-746, 1977.

[3] L. J. Grubka and K. M. Bobba, "Heat transfer characteristics of a continuous stretching surface with variable temperature," Journal of Heat Transfer, vol. 107, no. 1, pp. 248-250, 1985.

[4] M. E. Ali, "Heat transfer characteristics of a continuous stretching surface," Warme Stoffubertrag, vol. 29, no. 4, pp. 227-234, 1994.

[5] K. Vajravelu, "Convection heat transfer at a stretching sheet with suction or blowing," Journal of Mathematical Analysis and Applications, vol. 188, no. 3, pp. 1002-1011, 1994.

[6] I. Pop and T. Y. Na, "Unsteady flow past a stretching sheet," Mechanics Research Communications, vol. 23, no. 4, pp. 413-422, 1996.

[7] T. R. Mahapatra and A. S. Gupta, "Stagnation-point flow towards stretching surface," Canadian Journal of Chemical Engineering, vol. 81, no. 2, pp. 258-263, 2003.

[8] C. Y. Wang, "Liquid film on an unsteady stretching surface," Quarterly of Applied Mathematics, vol. 48, no. 4, pp. 601-610, 1990.

[9] H. I. Andersson, J. B. Aarseth, N. Braud, and B. S. Dandapat, "Flow of a power-law fluid film on an unsteady stretching surface," Journal of Non-Newtonian Fluid Mechanics, vol. 62, no. 1, pp. 1-8, 1996.

[10] H. I. Andersson, J. B. Aarseth, and B. S. Dandapat, "Heat transfer in a liquid film on an unsteady stretching surface," International Journal of Heat and Mass Transfer, vol. 43, no. 1, pp. 69-74, 2000.

[11] B. S. Dandapat, B. Santra, and H. I. Andersson, "Thermocapillarity in a liquid film on an unsteady stretching surface," International Journal of Heat and Mass Transfer, vol. 46, no. 16, pp. 3009-3015, 2003.

[12] C. H. Chen, "Heat transfer in a power-law fluid film over a unsteady stretching sheet," Heat and Mass Transfer, vol. 39, no. 8-9, pp. 791-796, 2003.

[13] C. Wang, "Analytic solutions for a liquid film on an unsteady stretching surface," Heat and Mass Transfer, vol. 42, no. 8, pp. 759-766, 2006.

[14] C. Wang and I. Pop, "Analysis of the flow of a power-law fluid film on an unsteady stretching surface by means of homotopy analysis method," Journal of Non-Newtonian Fluid Mechanics, vol. 138, no. 2-3, pp. 161-172, 2006.

[15] B. S. Dandapat, B. Santra, and K. Vajravelu, "The effects of variable fluid properties and thermocapillarity on the flow of a thin film on an unsteady stretching sheet," International Journal of Heat and Mass Transfer, vol. 50, no. 5-6, pp. 991-996, 2007.

[16] M. A. A. Mahmoud and A. M. Megahed, "MHD flow and heat transfer in a non-Newtonian liquid film over an unsteady stretching sheet with variable fluid properties," Canadian Journal of Physics, vol. 87, no. 10, pp. 1065-1071, 2009.

[17] N. F. M. Noor and I. Hashim, "Thermocapillarity and magnetic field effects in a thin liquid film on an unsteady stretching surface," International Journal of Heat and Mass Transfer, vol. 53, no. 9-10, pp. 2044-2051, 2010.

[18] M. Abd El-Aziz, "Flow and heat transfer over an unsteady stretching surface with Hall effect," Meccanica, vol. 45, no. 1, pp. 97-109, 2010.

[19] R. C. Aziz, I. Hashim, and A. K. Alomari, "Thin film flow and heat transfer on an unsteady stretching sheet with internal heating," Meccanica, vol. 46, no. 2, pp. 349-357, 2011.

[20] A. M. Megahed, "HPM for the slip velocity effect on a liquid film over an unsteady stretching surface with variable heat flux," European Physical Journal Plus, vol. 126, no. 9, article 82, 2011. 
[21] I.-C. Liu and A. M. Megahed, "Numerical study for the flow and heat transfer in a thin liquid film over an unsteady stretching sheet with variable fluid properties in the presence of thermal radiation," Journal of Mechanics, vol. 28, pp. 559-565, 2012.

[22] A. Raptis, "Flow of a micropolar fluid past a continuously moving plate by the presence of radiation," International Journal of Heat and Mass Transfer, vol. 41, no. 18, pp. 2865-2866, 1998.

[23] A. Raptis, "Radiation and viscoelastic flow," International Communications in Heat and Mass Transfer, vol. 26, no. 6, pp. 889-895, 1999.

[24] B. S. Dandapat and G. C. Layek, "Spin coating in the presence of a transverse magnetic field and nonuniform rotation: a numerical study," Journal of Physics D, vol. 32, no. 19, pp. 2483-2491, 1999. 


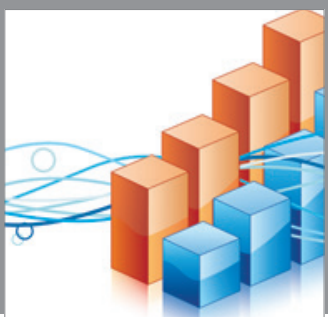

Advances in

Operations Research

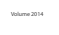

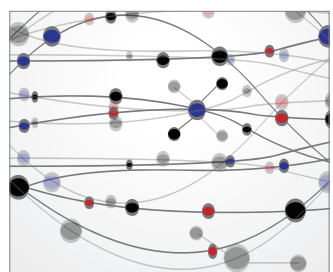

\section{The Scientific} World Journal
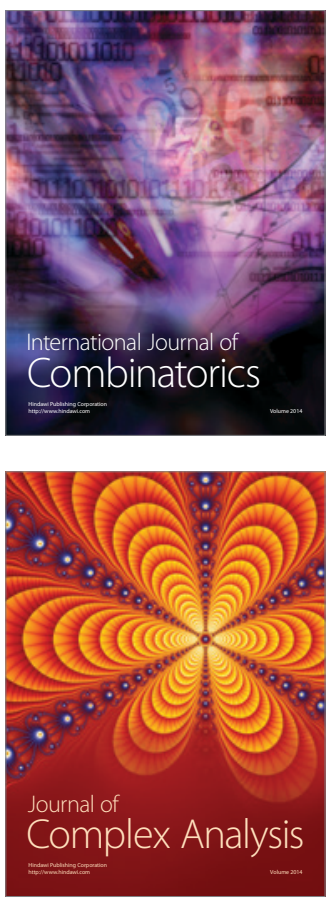

International Journal of

Mathematics and

Mathematical

Sciences
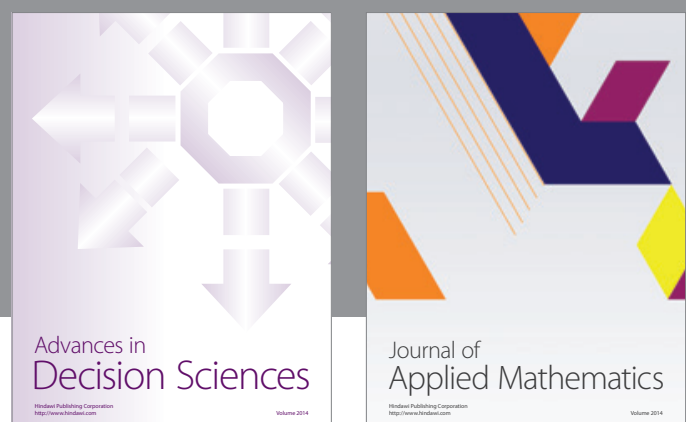

Journal of

Applied Mathematics
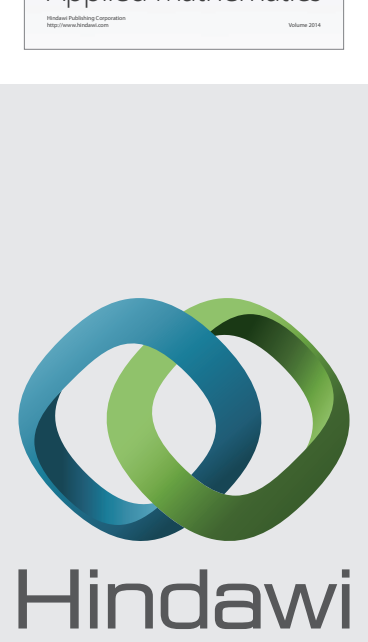

Submit your manuscripts at http://www.hindawi.com
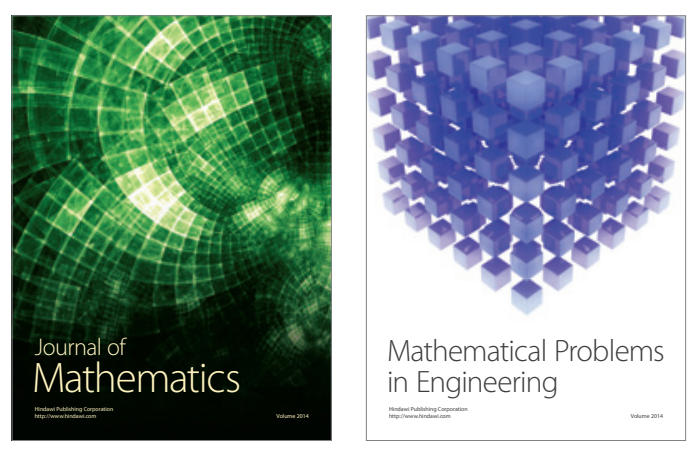

Mathematical Problems in Engineering
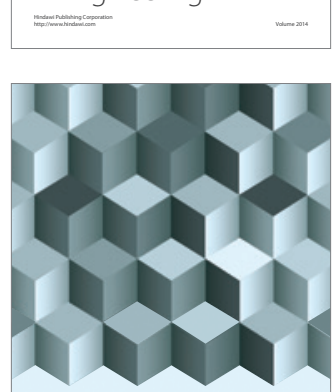

Journal of

Function Spaces
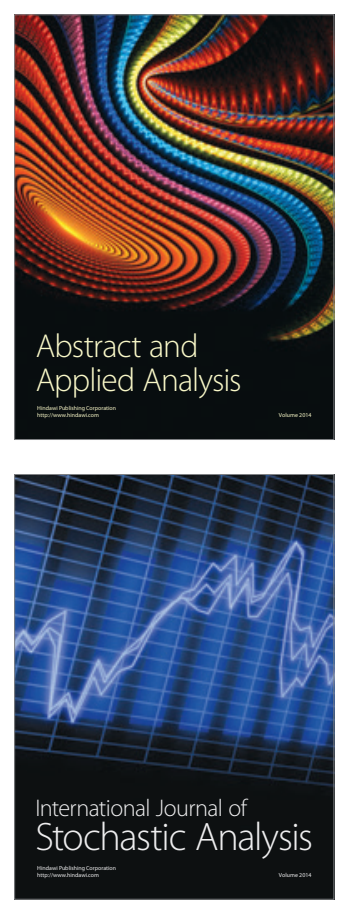

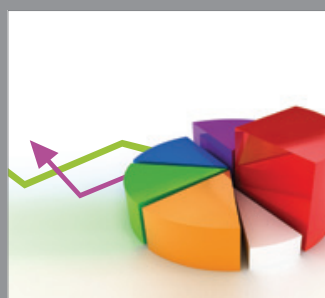

ournal of

Probability and Statistics

Promensencen
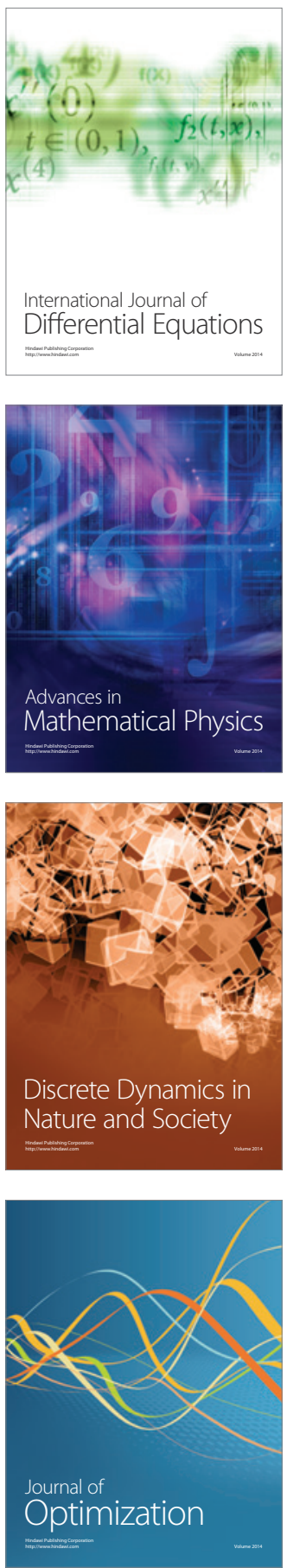